\title{
勘察技术在岩土工程勘察中的应用
}

朱斌

荆州市城市规划设计研究院，湖北 荆州 434000

[摘要] 随着社会的进步我国在科研上有了新的发展, 目前在岩土工程上的勘察应用我国也有了新的具体操作, 岩土勘察技术 也是目前我国在勘察技术上的主要发展对象。岩土勘察技术对于岩石工程来说是研究的前提需求, 岩土勘察是对岩土层运用 一定的技术手段来分析这块岩土层的特性和价值意义。岩土勘察工作为勘察建筑工作的进行做一些前期的准备。据目前情况 来看, 我国的岩土勘察技术存在了一些问题, 导致在工程进行勘察的时候出现一些可以避免的问题, 所以此文章就是以勘察 技术在勘察岩土工程上的运用展开具体研究。

[关键词] 勘察技术; 岩土工程勘察; 应用分析; 具体改善方法

DOI: $10.33142 /$ ec.v4i1.3235 中图分类号：TU195 文献标识码：A

\section{Application of Investigation Technology in Geotechnical Engineering Investigation}

ZHU bin

Urban Planning and Design Institute of Jingzhou, Jingzhou, Hubei, 434000, China

\begin{abstract}
With the progress of society, there is a new development in scientific research in our country. At present, our country also has a new specific operation in the application of geotechnical engineering investigation and geotechnical investigation technology is also the main development object in our country. Geotechnical investigation technology for rock engineering is the premise of research needs, geotechnical investigation is to use certain technical means to analyze the characteristics and value of this rock and soil layer. The geotechnical investigation makes some preparations for the construction investigation. According to the current situation, there are some problems in our country's geotechnical investigation technology, which lead to some avoidable problems in the engineering investigation, so this article is to carry out specific research on the application of investigation technology in geotechnical engineering investigation.
\end{abstract} Keywords: investigation technology; geotechnical engineering investigation; application analysis; specific improvement methods

引言

我国的工业建造问题相比之前已经有了很高的提升技术，不管是在勘察方法还是勘察技术上相比之前的传统技术 都随着社会科学技术的进步有所提升。我国的勘察技术是目前工业进行建造的前期发展技术, 如果勘察技术没有一个 很好的技术应用后期的建造工程开展就会出现很多问题。例如: 岩土层技术前期开展不顺利, 类型特性了解不清晰等 等。在开展过程中可能会出现建造工程出现错误、技术不过关等问题就说明这是类型特性不了解清晰的问题。所以在 岩土层勘察问题上就需要加强重视度岩土层勘察应用的相关技术目前应用范围很广, 所以对于社会发展来说勘察技术 的提高是有着极大的利用技术的。但是目前勘察技术人员的数量上还是较为少的, 在此方面国家、政府和社会要积极 的采取鼓励措施，通过加强技术人员的薪资待遇等方式来推广技术人员这一工作的宣传。

\section{1 研究勘察技术在岩土工程勘察中应用的现实意义}

实验研究显示, 我国对建筑行业的重视, 促使了科技人员对勘察技术水平的提高, 这也保障了我国在岩土工程技 术上的可持续发展性。由于国家的重视, 相关的勘察技术、工艺以及方法都被发掘了出来被用到岩土勘察工程上。由 于光照和气候等多种因素的影响, 导致了各个不同地区的岩土环境不一样, 而各个地区对于岩土勘察在岩土工程技术 上的要求也是不太一样的。所以为了提高岩土工程的施工进度, 需要技术人员在不同地区对已建成的建筑工程项目的 勘察技术情况进行整理, 总结出不同地区在岩土勘察过程中出现的问题并着手进行解答。而在未建成的项目中, 就能 根据总结出的问题和当地的地质做出判断进而提高工作效率, 也能够对实际的和预期的进行完善。在地质勘查准确地 情况下, 对工程进度的控制严格要求, 以此为条件, 来达到事半功倍的预期目标。研究勘察技术在岩土工程上的勘察 意义在于让勘察人员了解到勘察技术的具体作用, 增加自我肯定性, 进而帮助勘察人员提高自己的专业能力。

\section{2 岩土工程勘察技术的发展有什么目的?}

岩土工程的开展中主要对于岩土层进行采取之后, 结合要建造的建筑工程具体内容后, 分析采取样品的岩土层适 
不适合施工的建筑物。在研究过程中要理性的结合地基的具体稳定性、均匀度、承载力等方式来分析岩土层的适合度。 而岩土工程中对于勘察技术的目的是什么? 主要以保障建筑的顺利进行同时在建筑工程可以更好的方式建造在研究土 地上。例如: 在施工过程中对于岩土层的研究会出现一些研究参数, 根据研究参数我们可以得到一些基本的信息, 通 过信息联想到建筑工程过程中会出现的一些问题。并且岩土层不仅仅可以对于土地进行勘察, 对于在水里的施工工程 同时可以有一定的勘察。在岩土层进行采样分析时, 如果研究参数不是很准确就会出现在施工上出现因为参数而出现 的误差在水上建造工程会相比陆地上更加的有风险, 可能一个不小心就会出现很大的问题。由于水中的压力很大, 水 的流速又具有不可控性, 就非常容易出现问题。但是岩土研究技术可以在岩土工程中勘察出来的结果根据具体情况放 在施工建筑过程中, 抓住勘察出的容易影响建筑工程的问题重视起来避免问题得出现影响建筑工程的开展, 达到一个 基本保障的作用。所以总的来说, 在岩土工程上利用勘察工作还是有很多的好处。虽然在建造过程中避免不了问题的 出现, 但是对于大问题, 高危险等的事情应该有一个防御的作用。

\section{3 岩土工程勘察中勘察技术的应用控制策略}

\section{1 取样勘察技术}

岩土勘察中最重要的一步就是取样。而在实际工地中的手段有三种: 钻探工程、坑探工程和地球物理勘探, 这些 都需要结合本地的地质环境和当地的历史文化判断当地的地质类型, 进而选择钻探技术。钻探工程是最普遍的一种取 样技术, 能用于各种岩石环境中, 但是由于它会误判, 所以又被称为 “半直接” 勘探技术手段。勘探工程中勘探技术 人员可以通过这种技术找到地质细节。相比于钻探和坑探工程, 物理勘探则是一种间接的手段, 它具有立体性还能利 用电脑做出相关模型来实体化, 更能清晰的看到深层地质的情况所以成本也是比较低, 速度快。它还能解决手绘不出 来的且无法解决的问题。这就需要技术人员在掌握技术规律的同时还要熟练掌握电脑的相关模型的技术, 从而来加快 进程, 但是在取样过程中是需要勘察人员极具小心的, 一个不小心很有可能采集工作就需要重新开展。同时在采样过 程中要理性的分析当时的具体环境, 要采集到最普遍条件之下的样品, 避免在研究过程中发生误差。

\section{2 原位测试与室内试验技术}

原位测试和室内检验技术是对岩土勘察在岩土工程设计中的勘察工作方法之一, 它的检验结果对建设方面有很大 的帮助。所以, 在岩土工程技术中岩土勘察地质结构的稳定与否及其相关问题的分析。原位测试实验是在岩土原来所 处的位置上或基本上在原位状态和应力条件下对岩土性质进行的测试所以在测试时, 技术人员要在对准原位状态和应 力条件允许的前提下对当地的岩土情况进行分析, 再对性质进行检测以保证获取的数据和实际情况一致。这种方式操 作简单, 周期性短, 还能简单的对基础结构进行勘探, 对造价的成本有所控制。所以要重视对相关勘探技术人员的培 养, 对技术人员要严格要求, 这样才能为勘察工作有所帮助。在管理技术人员上, 因为技术人员的专业水平与勘察工 程的开展息息相关, 所以从专业人员的专业能力上需要定期的进行考核。通过考核之后的人员再根据平时的能力进行 分配任务，考核不过关的人员要设定相关的措施来帮助人员进行专业能力的提高。

\section{3 物理勘察技术}

地球物理勘探技术又称为 “物探”, 是利用不同的物理性质的岩层和煤层对地球物理场所产生的异常来寻找矿体, 圈定含煤区域、推断地质构造及解决其他地质问题的一种技术手段。常用的物理手段有重力勘探、磁法勘探、电法勘 探、地震勘探。在勘探信息处理的过程中, 要注意统一电阻率。同时, 还要将前面整理出来的地质资料利用起来, 然 后再分析对声波测孔与现场钻孔的差别, 再结合原来得到的地质信息进行对比。这样一来, 就可以得出相关的物理性 质参数。物理勘察技术相比之前的传统技术在技术上是一个很高的提升, 在对勘察对象尽可能的不破坏之下利用物理 技术可以得到勘察物的一系列相关信息。物理勘察技术总体上来说是最适合应用在有关水方面的勘察工作, 显而易见 水上的勘察工作会相比陆地更难勘察, 要用到的勘察技术很复杂且需要考虑的因素也有很多, 不仅如此勘察技术中的 电钻技术是没有办法应用的, 所以在水方面的勘察技术上物理勘察技术是很需要的。如果要提高岩土勘察在岩土工程 技术中的准确性，就需要对相关数据进行准确的记录，对准确的记录分析后再开展物理勘察技术。

\section{4 电钻技术}

电钻技术在勘察技术中是应用十分广泛的一项技术，在岩土采取过程中对于深层的岩石技术人员往往会使用电钻、 电锤等技术进行开采。电钻技术的开采减少了人力的使用, 同时对于工程的进速也有着很高的加快作用。所以说不管 是在技术使用上还是在人力上都有着对于勘察作用很高的帮助作用。电钻技术相比之前的传统勘察技术是一个崭新的 
发展, 虽然省力不少但是对于岩石的破坏性也是很高的, 所以在电钻技术方面目前还是有很大的提升空间去改善的。 目前运用最广泛的就是电钻的取芯技术, 电钻钻探技术可以与红外线相结合借助科学技术清晰的找到软土的具体位置, 同时可以清晰的看到软土的颜色、形态、包含度、内容量等等基本情况。所以总的来说, 电钻技术是最基本的勘察技 术，也是在勘察工作实施工程从始到终一直使用的技术。

\section{4 勘察技术在岩土工程勘察中的应用现状}

岩土勘察是一项全面性地、复杂性的地质调查工作, 采用各种侦查技术对当地岩土进行分析整理。岩土勘察部分 最重要的一项就是滑坡, 滑坡所产生的一些自然灾害导致的地质破坏, 给岩土工程建设增加了难度。而如今, 对于各 行业逐步完善, 工程建筑行业也逐渐趋向复杂性、精确性, 对所处地区的岩土勘察工作必须做到全面覆盖的同时还要 有准确性。建筑工程行业就是为了人们的生活需求而开设的。所以, 满足广大人民群众的需求的同时还要保证人们的 安全, 相关技术人员要对滑坡所处的地带进行分析, 要实时监测和控制滑坡导致的地质灾害。再进行相关检验的应用 之后才能获得更深一步的信息数据, 方能为以后做打算, 虽然勘察技术已经逐步成熟, 但是在这些地区获取数据时, 还是很容易受到滑坡的实际情况的影响, 在勘察工作方面工作效率低而且价格还高。所以这就需要技术人员想办法将 探地雷达法与高密度电阻率法用到实际的勘察工作中。探地雷达法, 就是根据广播电磁波探测技术, 来对地底介质分 布情况进行确定。这类技术主要就是用到复杂地形中, 而滑坡地带就是其中之一。这种技术仪器有很多优点。比如, 具有高精度、造价低, 重要的是工作效率高。但是也有很多不足。比如, 局限性大: 不能用到各类岩石中, 而且还需 要很多仪器来精确判断信息的准确性。

\section{5 结语}

由上述讲述可知, 岩土勘察在岩土工程技术中是很重要的, 但是在技术手段成熟的过程中面临的情况也很复杂。 而且技术手段和方法都受外界环境和当地岩土地质的影响。所以在岩土勘察的过程中需要提高技术手段和相关技术人 员的培养, 进而可以为建设提供准确的信息数据。建设者也要对不同地区的勘察情况和地质情况进行分析积累经验, 为以后降低环境对建设的影响。随着目前新社会的发展情况, 科学技术都有一个很高的发展前景, 越来越多的科学信 息技术运用到工程建造上, 勘察技术也应该随着社会的进度有所提高。不管是对这一行来说还是整个社会来说, 都是 很重要的。所以以上就是对勘察岩土层做出的具体内容分析, 希望对于与之有关的工作人员有好的帮助作用。

\section{[参考文献]}

[1] 张衍. 岩土工程勘察设计和施工过程中的水文地质问题分析 [J]. 工程建设与设计, 2018(2): 87-88.

[2]叶章.瞬态面波及地震影像在岩土工程勘察中的应用 $[\mathrm{J}]$. 山西建筑, 2019, 44 (27): 66-68.

[3]义家吉.颜历勘查技术方法在岩土工程勘察中的应用 [J]. 居业,2017 (118) : 11-13.

作者简介: 朱斌( 1984.10-) 男, 西安科技大学, 地质工程, 现在单位: 荆州市城市规划设计研究院, 工程师。 